http://jmscr.igmpublication.org/home/ ISSN (e)-2347-176x ISSN (p) 2455-0450 crossref DOI: https://dx.doi.org/10.18535/jmscr/v8i4.30

\title{
A Correlation Study between Non-Alcoholic Fatty Liver Disease and Severity of Coronary Artery Disease
}

\author{
Authors \\ Dr Seba Brata Jana ${ }^{1}$, Dr Kaushik Paul ${ }^{2 *}$, Dr Bidyut Roy ${ }^{3}$, Dr Sankar Chandra Mandal ${ }^{4}$ \\ ${ }^{1,3}$ SR, IPGMER and SSKM Hospital Kolkata \\ ${ }^{2}$ RMO, IPGMER and SSKM Hospital Kolkata \\ ${ }^{4}$ Professor, IPGMER and SSKM Hospital Kolkata \\ *Corresponding Author \\ Dr Kaushik Paul
}

SSKM Doctor's Hostel, IPGMER And SSKM Hospital, Kolkata 700020, India

\section{Introduction}

According to the World Health Organization, the most common cause of death worldwide is cardiovascular disease (CVD) of which Ischaemic Heart disease stands high in the list. Risk factors of IHD can be non-modifiable \& modifiable factors The Modifiable factors are hypertension, smoking, dyslipidemia, diabetes, obesity, sedentary lifestyle \& stress which always are the target areas for reducing the morbidity and mortality related to IHD. Non-alcoholic Fatty Liver Disease (NAFLD) may be considered a modifiable risk factor for IHD. Recently emerging evidences suggests that the incidence of Nonalcoholic Fatty Liver Disease (NAFLD) is high among patients of Coronary Artery Disease(CAD) in comparison to general population, and this association is independent of other metabolic risk factors of IHD, and cardiovascular disease is the most important cause of death in patients suffering from NAFLD ${ }^{(1,2)}$. So identifying NAFLD in early stage and proper management may help in reducing the future risk of $\mathrm{CAD}$ and thus reducing the morbidity and mortality related to CAD. There are evidences in literature that shows the association between NAFLD and Coronary Artery Disease (CAD) but no enough evidences so far to prove causal relationship. This study we conducted to estimate the prevalence of NAFLD as diagnosed by ultrasonographic examination of the liver and to assess the association between NAFLD and CAD and to find if there is any correlation between severity of CAD with NAFLD. We used coronary angiography to assess the presence of CAD. While assessing coronary angiography we calculated SYNTAX score to estimate anatomical complexity of CAD and as a marker of severity of CAD. In Patients with NAFLD, we also assessed severity by grading of fatty liver with USG criteria and by stiffness of liver using Fibroscan. We tried to found out any association with grades of severity fatty liver with severity of CAD. 


\section{Aims and Objectives}

1) To assess the correlation between coronary artery disease and non-alcoholic fatty liver disease.

2) To find if severity of coronary artery disease is related to severity of hepatic steatosis and hepatic fibrosis

\section{Materials and Methods}

It is a Cross sectional observational type of study was conducted in the Department of Cardiology in a tertiary care hospital in eastern india. Male and female patients of acute coronary syndrome (including STEMI, NSTEMI or UA) and stable coronary artery disease with indication for revascularization who were Admitted for Coronary angiography in our institution and who gave consent for this study were included. During the period of February 2018 to October 2019, the study was conducted. All patients with age more than 18 years, previously and recently diagnosed CAD patients who have indication for $\mathrm{CAG}+/-$ revascularization, who was Admitted in department of Cardiology and consented for the study were included in this study. Patients who are unwilling to participate, chronic alcoholic, who had chronic liver disease and HBV,HCV positive were excluded from this study patients who fulfil the inclusion criteria were selected for the study. At first detailed informed consent was taken for coronary angiography (CAG) and for being included in the study. CAG was done and severity of CAD was assessed by calculating SYNTAX score using online calculator. Thereafter presence of NAFLD was assessed by ultrasonography and was graded as fatty liver grade $0,1,2$, and 3 using diagnostic criteria. Liver stiffness was assessed by Fibroscan and severity was expressed as $\mathrm{kPa}$ score. For statistical analysis data were entered into a Microsoft excel spreadsheet and then analyzed by SPSS.

\section{Results}

Total 300 patients were included in this study. All the patients had coronary artery disease (CAD) either in the form of acute coronary syndrome or stable angina with indication for revascularization. Patients were admitted for coronary angiography +/- revascularization and subsequently undergone biochemical tests, ultrasonograpgy to look for fatty liver and fibroscan to define liver stiffness. Mean age of patients were 55.15 years. $28.3 \%$ of total study population was female and $71.7 \%$ were male with male to female ratio of around 2.5:1. We found NAFLD was present in 55\% of patients of which $43.0 \%$ patients had grade 1 Fatty Liver and $12.0 \%$ patients had grade 2 Fatty Liver and $45.0 \%$ patients had grade 0 or No Fatty Liver. The mean BMI (mean \pm s.d.) of patients of CAD without fatty liver was $24.0310 \pm 1.9342$, and that of grade 1 fatty liver patients was $25.4248 \pm$ 2.1640 and grade 2 fatty liver patients was $26.2072 \pm 2.5087$. Difference of mean BMI with three grades was statistically significant $(p<0.0001)$. That means patients of CAD with associated NAFLD had higher mean BMI than patients without NAFLD. The mean Waist Circumference (mean \pm s.d.) of patients of CAD without fatty liver was $84.0148 \pm 6.3399$, and that of patients with grade 1fatty liver was $93.9147 \pm$ 7.3240 and grade 2 fatty liver patients was $97.0278 \pm 8.2928$. Difference of mean Waist Circumference with three grades was statistically significant $(p<0.0001)$. That means patients of CAD with associated NAFLD had higher mean Waist Circumference than patients without NAFLD. The mean Triglyceride level $(\mathrm{mg} / \mathrm{dl})$ (mean \pm s.d.) of patients of CAD without fatty liver was $157.1556 \pm 23.4313 \mathrm{mg} / \mathrm{dl}$, and that of patients with grade 1 fatty liver was $167.3876 \pm$ $26.2885 \mathrm{mg} / \mathrm{dl}$ and grade 2 fatty liver patients was $160.3056 \pm 23.4214 \mathrm{mg} / \mathrm{dl}$. Difference of mean Triglyceride level $(\mathrm{mg} / \mathrm{dl})$ with three grades was statistically significant $(\mathrm{p}=0.0036)$. That indicates patients of CAD The mean LDL level $(\mathrm{mg} / \mathrm{dl})$ (mean \pm s.d.) of patients of CAD without fatty liver was $109.5111 \pm 88.9580 \mathrm{mg} / \mathrm{dl}$, and that of patients with grade 1 fatty liver was $119.9845 \pm$ $21.1915 \mathrm{mg} / \mathrm{dl}$ and grade 2 fatty liver patients was $128.1667 \pm 13.8863 \mathrm{mg} / \mathrm{dl}$. There was no 
statistically significant Difference between the mean LDL level (mg/dl) among the three grades of fatty liver patients $(\mathrm{p}=0.1805)$. With associated NAFLD had higher mean Triglyceride level than patients without NAFLD. $36.7 \%$ of total study population was diabetic and $71.7 \%$ were nondiabetic. Among the non-diabetic sub-population $49.5 \%$ had grade 0 or no fatty liver and $50.5 \%$ had fatty liver. The prevalence of fatty liver among diabetic patients was higher $62.8 \%$ (grade 1 $45.5 \%$, grade $2-17.3 \%$ ). NAFLD was more prevalent in diabetic patients with CAD than in non-diabetic population. Prevalence of Metabolic syndrome in our study population with all patients having CAD was $54.33 \%$. Among them fatty liver was present in $69.9 \%$ of patients. Sub population which does not fulfil criteria for metabolic syndrome was $45.67 \%$. The prevalence of NAFLD among patients without metabolic syndrome was $37.2 \%$, of which 31.45 had grade 1 fatty liver and $5.8 \%$ had grade 2 fatty liver. Fibroscan determines the stiffness of liver. When we compared fatty liver with fibroscan value, we found that The mean Fibroscan ( $\mathrm{kPa})$ (mean \pm s.d.) of patients with grade 0 or no fatty liver was $3.7548 \pm .7162 \mathrm{kPa}$, grade 1 patients was $5.3248 \pm$ $1.8376 \mathrm{kPa}$ and grade 2 patients was $6.7222 \pm$ $2.2104 \mathrm{kPa}$. Difference of mean Fibroscan $(\mathrm{kPa})$ among the three grades was statistically significant $(\mathrm{p}<0.0001)$ (Table 1) That indicates patients with higher grade of fatty liver have higher fibroscan values. We assessed severity of CAD by angiographic criteria using SYNTAX score and tried to find any correlation with liver stiffness by comparing it with fibroscan values. We found a positive correlation between FIBROSCAN $(\mathrm{kPa})$ and SYNTAX SCORE and that was statistically significant (Figure 1). That indicates patients with $\mathrm{CAD}$ are tend to have higher liver stiffness or higher fibroscan values than patients without CAD. When we tried to correlate severity of CAD with severity of NAFLD by grades of fatty liver, however we did not find any statistically significant corelation between fatty liver and syntax score

\section{Discussions}

NAFLD affects about $25 \%-30 \%$ of the adult population in Western countries, but its prevalence is increasing in developing countries (3) A recent meta-analysis with a total number of $8,515,431$ patients reported that the global prevalence of NAFLD is 25.24 There is paucity of published literature regarding the prevalence of NAFLD in India, with most studies describing a small number of patients. Epidemiological studies suggest prevalence of NAFLD in around 9-32\% of general population with maximum prevalence in those between 40 and 50 years of age ${ }^{(4,5)}$. The lowest prevalence of $9 \%$ was reported from the rural part of West Bengal (East India), whereas the highest prevalence of $32 \%$ was reported from the urban part of southern India. In our present study, we tried to evaluate prevalence of NAFLD among CAD patients and thus to find any correlation between CAD and NAFLD. In our total study population all of whom had CAD, we found prevalence of NAFLD was 55\% (with $43 \%$ having grade 1 fatty liver and $12 \%$ having grade 2 fatty liver) which is much higher in comparison to NAFLD prevalence in general population. That indicates a positive correlation between NAFLD and CAD as shown in various other studies. Almost similar result was found in the study by Tahmineh Tavakoli et al. ${ }^{(6)}$ in Iran where prevalence of NAFLD in CAD patients was $59.1 \%$ with different grades including $46.2 \%$ Grade I and $12.1 \%$ grade II. Khwaja Saifullah Zafar et al. in their study showed the prevalence of NAFLD was $46 \%$ among patients with CAD. A similar result was there in the study of Atoosa Adibi et al, where prevalence of NAFLD was much higher in patients with $\mathrm{CAD}$ than in general population.

There was a concern discussed in literature whether the association between NAFLD and CAD is a real correlation or it is due to metabolic syndrome and different metabolic factors commonly associated with both the condition. However it was proved in various studies that NAFLD is associated with CAD independent of 
metabolic factors. We also found similar result in our study. $45.67 \%$ Patients of total study population with CAD did not have metabolic syndrome, and prevalence of NAFLD among them was $37.2 \%$. In our study we tried to found out if severity of CAD can be correlated with ultrasonographic severity grades of fatty liver and severity liver stiffness measured by fibroscan. We found a positive correlation between FIBROSCAN (kPa) and SYNTAX SCORE and that was statistically significant. That indicates patients with CAD are tend to have higher liver stiffness or higher fibroscan values than patients without CAD. Earlier studies assessed severity of CAD by coronary artery calcium score (CAC). Kim et al. in their study showed the presence of CAC (score $>0$ ) was significantly associated with NAFLD. Increasing CAC scores $(0,<10,10-100$, $\geq 100$ ) were associated with higher prevalence of NAFLD. However despite confirming the known observational association of liver fat content and NAFLD with CAD, none of the study found any causal relationship between high liver fat content and CAD. Similarly in our study we were also not able to establish causal relationship between NAFLD and CAD but a positive correlation between liver stiffness indicated by high fibroscan value and severity of CAD as indicated by SYNTAX score value was established in our study for the first time in literature.

\section{Conclusions}

So the cross sectional observational study showed that NAFLD is associated with CAD as evidenced by increased prevalence of fatty liver in patients of CAD in comparison to general population. This association was independent of other metabolic factors associated with both the condition as the prevalence of NAFLD was higher than normal population even in the sub-population without features of metabolic syndrome. When compared between severity of NAFLD with severity of $\mathrm{CAD}$, there was a positive correlation between severity of liver stiffness by Fibroscan value with SYNTAX score value as a measure of severity of
CAD. However no correlation was observed between grades of fatty liver and severity of CAD. Whether there is any causal relationship between NAFLD and CAD yet to be established.

\section{Limitations}

It was a single centre cross sectional observational study. Ultrasonography to diagnose fatty liver was performed by different operator at different times, so there may be observer bias. The gold standard for diagnosis of fatty liver is histo-pathological examination by liver biopsy; however we used USG considering the invasive nature of liver biopsy.

Keywords: Non Alcoholic Fatty Liver Disease, Coronary Artery Disease

There was no conflicts of interest of any authors, no research support

\section{References}

1. Lonardo A, Sookoian S, Pirola CJ, Targher G. Non-alcoholic fatty liver disease and risk of cardiovascular disease. Metabolism. 2016;65(8):1136-50.

2. Ballestri S, Lonardo A, Bonapace S, Byrne CD, Loria P, Targher G. Risk of cardiovascular, cardiac and arrhythmic complications in patients with nonalcoholic fatty liver disease. World $J$ Gastroenterol. 2014;20(7):1724-45.

3. Younossi Z, Anstee Q, Marietti M, Hardy T, Henry L, Eslam M et al. Global burden of NAFLD and NASH: trends, predictions, risk factors and prevention. Nature Reviews Gastroenterology \& Hepatology. 2017;15(1):11-20.

4. 4.. Das K, Das K, Mukherjee P, Ghosh A, Ghosh S, Mridha A et al. Nonobese population in a developing country has a high prevalence of nonalcoholic fatty liver and significant liver disease. Hepatology. 2010;51(5):1593-1602.

5. Amarapurkar D, Kamani P, Patel N, Gupte P, Kumar P, Agal S et al. Prevalence of 
non-alcoholic fatty liver disease:

population based study. Annals of Hepatology. 2007;6(3):161-163.

6. Tahmineh Tavakoli, Toba Kazemi, Homa

Mollaei, Fatemeh Salmani, Samira

Saghafi, Ensiah Sadat Mousavi, Mahyar

Mohamadifard and Gholamreza

Sharifzadeh. The Relationship Between the Prevalence of Non-Alcoholic Fatty Liver Disease (NAFLD) with the Severity of Coronary Artery Disease (CAD) in Patients Undergoing Coronary Artery Angiography: A Cross- Sectional Study. Iran Red Crescent Med J. 2018 June; 20(6):e58369. 